\title{
Effects of Molecular Weight of Nylon 6 on the Structure and Properties of Nylon 6/Poly (p-phenyleneterephthalamide) Molecular Composites
}

\author{
Shigeyuki Ueta, Takeya Sakamoto, and Motowo Takayanagi \\ Department of Industrial Chemistry, Faculty of Engineering, Kyushu Sangyo University, \\ Matsukadai 2-3-1, Higashi-ku, Fukuoka 813, Japan
}

(Received June 8, 1992)

\begin{abstract}
The effects of molecular weight of nylon 6 on the phase separation of nylon 6 in the molecular composites of nylon 6/poly ( $p$-phenyleneterephthalamide) (PPTA) kept at $240^{\circ} \mathrm{C}$ and their mechanical properties related to molecular dispersity of PPTA have been studied on the coagulated and hot-pressed film. Crystallization of nylon 6 in the composites was studied by infrared spectroscopy, differential scanning calorimetry and X-ray diffraction. The crystallinity of nylon 6 was lost in the presence of PPTA when the PPTA content exceeds $50 \mathrm{wt} \%$ through all the range of molecular weight of nylon 6 . The $\mathrm{N}-\mathrm{H}$ stretching bands characteristic of PPTA and nylon 6 united to show a sharp single peak for the hot-pressed molecular composite for nylon 6 with $M W=2.6 \times 10^{5}$, indicating the interruption of aggregation of PPTA molecules. The tensile modulus and strength were monotonously increased with increasing molecular weight of nylon 6 and weight fraction of PPTA in the composites. The improved dispersity of PPTA molecules gives outstanding mechanical properties to the molecular composite. The film strength was $170 \mathrm{MPa}$ and the modulus was $12 \mathrm{GPa}$ for the hot-pressed composite film of $10 \mathrm{wt} \%$ nylon 6 using its highest molecular weight.

KEY WORDS Poly( $p$-phenyleneterephthalamide) / Nylon $6 /$ Phase Separation / FTIR / Molecular Composite / Modulus / Strength /
\end{abstract}

The concept of molecular composite (MC) was for the first time in 1980 proposed by Takayanagi and his collaborators. ${ }^{1}$ Rigid rodlike molecules reinforce flexible coil-like molecules as a matrix. The principle of molecular composite is based on that of the macroscopic fiber-reinforced plastics. The expected advantages of MC are (1) the aspect ratio of rod molecules is large enough, (2) the ideal strength of covalent bonds in rod molecules is available, and (3) a homogeneous texture of blend is formed, which is most desirable for strength, being free from any defect introduced in a heterogeneous texture of blend. These advantages are realized only when molecularly homogeneous dispersion of rod molecules in flexible molecules is achieved. In reality, as far as unmodified rod molecules are employed, they have a strong tendency to associate in polymeric matrix to form a microfibrillar texture. Microfibrillar formation brings brittleness to the MC. The defects associated with heterogeneous texture are found at the both ends of fibrils and the microinterface between microfibril and matrix polymers. The most effective method to remove defects caused by micro-fibrillar texture is to use block copolymers ${ }^{1}$.

In this paper, the possibility of avoiding phase separation by using a matrix polymer with large molecular weight has been studied. The diffusion of rod molecules in a matrix of flexible molecules will be interrupted by increased internal viscosity of matrix coil molecules with high molecular weight. Kyu and others ${ }^{2}$ studied the miscibility in the MCs 
of PPTA/nylon 6 and PPTA/nylon 66 and found that the solvation of nylon 6 by PPTA was observed for the MC with PPTA content exceeding $70 \mathrm{wt} \%$. They explained the disappearance of nylon 6 crystal being ascribed to the cross-hydrogen bonding between both components. Phase separation at $240^{\circ} \mathrm{C}$, which temperature is above the melting point of nylon 6, was studied by them mainly on the basis of FTIR spectra in the regions of $\mathrm{N}-\mathrm{H}$ stretching and observed that the phase separation took place at $240^{\circ} \mathrm{C}$. They mentioned nothing about the effects of molecular weight of nylon 6. This paper reports that huge molecular weight of nylon 6 inhibits the phase separation at $240^{\circ} \mathrm{C}$.

\section{EXPERIMENTAL}

\section{Materials}

Poly ( $p$-phenyleneterephthalamide) (PPTA) used in this study has number average molecular weight of $5 \times 10^{4}$, which was obtained from Asahi Chemical Industry Company. Samples of nylon 6 (Ny6) with a wide range of molecular weight were presented from Toray company. The samples were specifically synthesized for this study, among which three kinds of nylon 6 were used: the number average molecular weight $(M W)$ of Ny6-A is $1.86 \times 10^{4}, M W$ of Ny6-B is $1.10 \times 10^{5}$, and $M W$ of Ny6-C is $2.60 \times 10^{5}$. Ny6-A and $\mathrm{B}$ were polymerized by conventional ringopening reaction of $\varepsilon$-caprolactam, and Ny6-C was synthesized by anion polymerization. The difference of molecular weight distribution was not taken into account in the discussion.

\section{Preparation of Molecular Composites}

The blend ratios of Ny6/PPTA (N/P) were $9 / 1,7 / 3,5 / 5$, and $1 / 9$. Both components were dissolved in $97 \%$ sulfuric acid to adjust the total concentration of both polymers to $2.8 \mathrm{wt} \%$. Use of three kinds of Ny6-A, B, and $\mathrm{C}$ gives 12 kinds of blend solutions.

The solution was sheared between two glass plates, coagulated in a plenty of water and further neutralized with a dilute aqueous ammonia solution. The film was dried on a Teflon sheet and hot-pressed at $240^{\circ} \mathrm{C}$ for $5 \mathrm{~min}$ at the pressure of $50-80 \mathrm{~kg} \mathrm{~cm}^{-2}$. The hot-press temperature, $240^{\circ} \mathrm{C}$, is reported by Kyu and others ${ }^{2}$ that the spinodal decomposition of MC takes place at this temperature. The film thickness was $3-6 \mu \mathrm{m}$.

\section{Characterization and Mechanical Testing}

Polarization microscopic observation of MC film was conducted under crossed polars. Infrared spectra were obtained in the range of $500-4300 \mathrm{~cm}^{-1}$ on a Nihonbunko FT/ IR-5000 spectrometer. A Rigaku DSC-8230 Differential Scanning Calorimeter was utilized with a nitrogen purge. Heating rate was undertaken at $10^{\circ} \mathrm{C} \mathrm{min}^{-1}$ to the temperature as high as $330^{\circ} \mathrm{C}$ and cooling rate was in a natural way. Alumina was used as the reference substance for temperature calibration. A Rigakudenki Geiger Flex RAD-IIIA X-ray diffractometer was employed for recording diffraction intensity curves for the films.

Tensile testing was conducted by using an Orientec Tensilon STM-T-50B tensile tester. The specimen size was $4 \mathrm{~mm}$ wide and $30 \mathrm{~mm}$ long. The load-elongation curves at room temperature were recorded with $200 \mathrm{~mm} \mathrm{~min}^{-1}$ chart speed and $5 \mathrm{~mm} \mathrm{~min}^{-1}$ loading rate with $2 \mathrm{~kg}$ maximum load.

\section{RESULTS AND DISCUSSION}

\section{Optical Microscopy}

Thin films of Ny6/PPTA used in this study were transparent and no clear sign of phaseseparated regions. Our purpose of research is to survey the phase separation in the molecular composite into microfibrillar PPTA phase and crystalline nylon 6 phase. Kyu and others ${ }^{2}$ have already observed that the spinodal decomposition takes place for the ratio of $\mathrm{N} / \mathrm{P}$ being larger than $7 / 3$ by heating the $\mathrm{MC}$ film at $240^{\circ} \mathrm{C}$ for $5 \mathrm{~min}$. The films in 

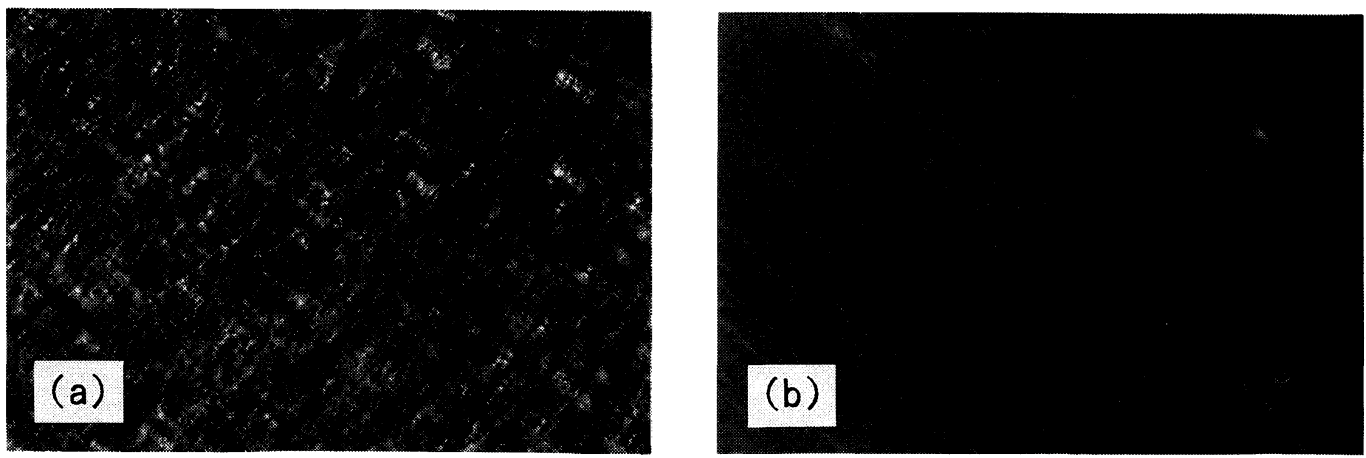

\section{$100 \mu \mathrm{m}$}

Figure 1. Polarization optical micrographs under crossed polars of the hot-pressed films of the molecular composite of $70 \mathrm{wt} \%$ of nylon $6(\mathrm{~N} / \mathrm{P}=7 / 3)$. (a) molecular weight of nylon 6 is $1.86 \times 10^{4}$; (b) $2.60 \times 10^{5}$.

this study were heated at $240^{\circ} \mathrm{C}$ under a hot press and the spinodal decomposition would have taken place, if the molecular weight of Ny6 in our study is comparable to that of their use.

Figures 1(a) and (b) show the polarization micrographs under crossed polars for the hotpressed films of $\mathrm{N} / \mathrm{P}=7 / 3$ (70 wt $\%$ nylon 6) using Ny6-A $\left(M W=1.86 \times 10^{4}\right)$ and Ny6-C $\left(M W=2.6 \times 10^{5}\right)$, respectively. Figure 1(a) shows birefringent image with striations crossing in $45^{\circ}$ directions to the crossed polars, indicating slight crystallization of the components. On the other hand, when Ny6-C is employed, detective birefringent image is absent, although the image is slightly lightened uniformly as a whole. This observation is different from the result reported by Kyu and others. $^{2}$ The increased molecular weight of nylon 6 interrupts the diffusion of PPTA molecules in the matrix to form a microfibrillar bundle, resulting in disappearance of birefringent image. As mentioned later, the crystallization of nylon 6 is also inhibited by an excess presence of PPTA, which effect also makes the image dark.

A similar result was obtained for $\mathrm{N} / \mathrm{P}=5 / 5$ using Ny6-C, but the birefringent image appeared for $\mathrm{N} / \mathrm{P}=9 / 1$ due to excess exis- tence of nylon 6. For the hot-pressed MCs of Ny6-A and B, the birefringent images were clearly detected for all the $\mathrm{N} / \mathrm{P}$ ratios, indicating the phase separation. Thus, the case of Ny6-C is a special case in which the phase separation is inhibited by heating at $240^{\circ} \mathrm{C}$ except $\mathrm{N} / \mathrm{P}=9 / 1$.

\section{FTIR}

FTIR scans were obtained in the range of $500-4300 \mathrm{~cm}^{-1}$ for the MCs of nylon 6/PPTA. The characteristic IR bands for PPTA and nylon 6 are given with respective band assignments in the literature. ${ }^{3-6}$

Figure 2 shows FTIR spectra in the regions of $\mathrm{N}-\mathrm{H}$ stretching for the compositions of $\mathrm{N} / \mathrm{P}=7 / 3$ and $5 / 5$ using Ny6-B. Absorption spectra 1 and 2 in Figure 2 are for the unannealed samples with the $\mathrm{N} / \mathrm{P}$ ratios of $7 / 3$ and $5 / 5$, respectively. According to the data in the literature the $\mathrm{N}-\mathrm{H}$ stretching band is located at $3329 \mathrm{~cm}^{-1}$ for PPTA and $3297 \mathrm{~cm}^{-1}$ for nylon 6 . The single sharp absorptions around $3300 \mathrm{~cm}^{-1}$ for the absorptions 1 and 2 are close to that of nylon 6 , but it is noticeable that the PPTA absorption can not be detected. Referring to the prediction of $\mathrm{Kyu}$ and others, ${ }^{2}$ a sharp single peak represents cross-hydrogen bonding be- 


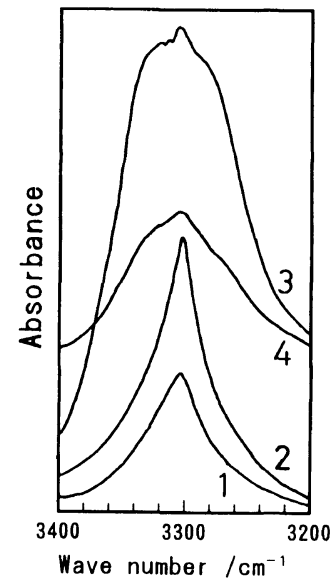

Figure 2. FTIR spectra in the regions of $\mathrm{N}-\mathrm{H}$ stretching for the unannealed and hot-pressed films of various compositions of nylon $6(\mathrm{~N}) / \mathrm{PPTA}(\mathrm{P})$ composites. Absorptions 1 and 2 are for the unannealed films of $\mathrm{N} / \mathrm{P}=7 / 3$ and $5 / 5$, respectively. Absorptions 3 and 4 are for the films hot-pressed at $240^{\circ} \mathrm{C}$ for $5 \mathrm{~min}$ with the $\mathrm{N} / \mathrm{P}$ ratios of $7 / 3$ and $5 / 5$

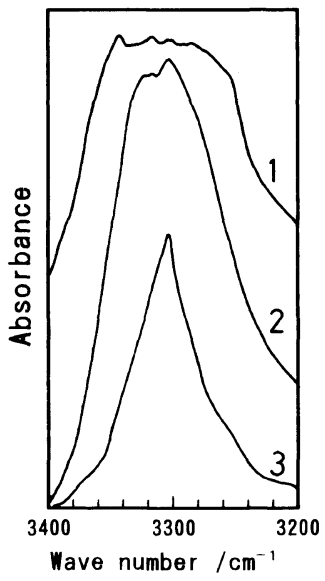

Figure 3. FTIR spectra in the regions of $\mathrm{N}-\mathrm{H}$ stretching for the hot-pressed films of $N / P=7 / 3$ with various molecular weights of nylon 6. Absorption 1 is for Ny6-A, absorption 2 is for Ny6-B and absorption 3 is for Ny6-C.

tween PPTA and nylon molecules. The heattreatment of the same samples by hot-pressing at $240^{\circ} \mathrm{C}$ gives the spectra 3 and 4 , which are for the $\mathrm{MC}$ of $7 / 3$ and $5 / 5$, respectively. The absorptions 3 and 4 are broadened and tend to split into $\mathrm{N}-\mathrm{H}$ stretching bands of PPTA and nylon 6. Phase separation into nylon 6

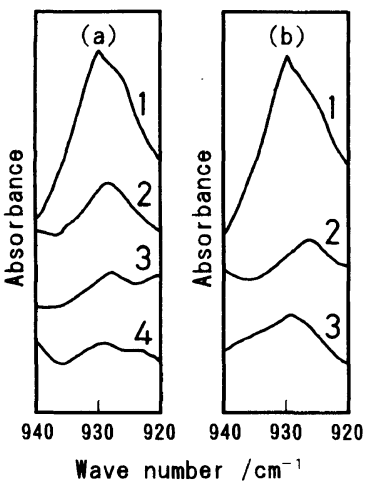

Figure 4(a) and (b). Infrared spectra of skeletal vibration of nylon 6 crystals in the composites with PPTA. (a) The effects of composition of $\mathrm{N} / \mathrm{P}=$ (1) $9 / 1$, (2) $7 / 3$, (3) $5 / 5$, and (4) $1 / 9$, using Ny6-A; (b) The effects of molecular weight of nylon 6 at $\mathrm{N} / \mathrm{P}=9 / 1$ with (1) Ny6-A, (2) Ny6-B, and (3) Ny6-C.

and PPTA by heat-treatment takes place: the characteristic absorptions of PPTA and nylon 6 coexist.

Figure 3 shows the absorption curves for the hot-pressed MC film of $\mathrm{N} / \mathrm{P}=7 / 3(70 \mathrm{wt} \%$ of nylon 6) in the same wave number range as in Figure 2. In Figure 3, the effect of molecular weight of nylon 6 is our main concern. Absorption curves 1 and 2 are for the hot-pressed $\mathrm{MC}$ films of $\mathrm{N} / \mathrm{P}=7 / 3$ using Ny6-A and Ny6-B, respectively. They show the broadness characteristic of the phase separation. When Ny6-C is used, a single sharp peak appears as shown in absorption 3, which indicates the cross-hydrogen bonding and the inhibition of phase separation. This result accords with the microscopic observation for the annealed MC film using Ny6-C, in which no birefringent image indicating phase separation has not been observed.

The $929 \mathrm{~cm}^{-1}$ band is assigned to nylon 6 crystal and $\mathrm{Kyu}$ and others ${ }^{2}$ reported that this band is observable up to $50 \mathrm{wt} \%$ of nylon $6(\mathrm{~N} / \mathrm{P}=5 / 5$ in our notation) and vanishes when the PPTA content exceeds $70 \mathrm{wt} \% \quad(\mathrm{~N} / \mathrm{P}=3 / 7$ in our notation $)$. It is observed that the $929 \mathrm{~cm}^{-1}$ band is largely depressed by annealing for $\mathrm{N} / \mathrm{P}=5 / 5$ even 
when the molecular weight of nylon 6 is as low as $1.86 \times 10^{4}$ (Ny6-A) as shown in Figure 4(a). This result shows that the crystallization of nylon 6 from the melt at $240^{\circ} \mathrm{C}$ is depressed in the presence of PPTA.

When the $N / P$ ratio is fixed at $9 / 1$, the $929 \mathrm{~cm}^{-1}$ band of nylon 6 crystal is observable independent of molecular weight of nylon 6. Figure 4(b) shows, however, that the peak intensity of absorption is monotonously decreased and broadened with increasing molecular weight. This means that the crystallization of nylon 6 is strongly interrupted by the dispersed state of PPTA molecules: PPTA molecules are more homogeneously dispersed in Ny6-C and do not allow for nylon 6 to freely crystallize.

The amide I and II bands of pure PPTA are located at 1655 and $1552 \mathrm{~cm}^{-1}$, respectively, while those of nylon 6 are 1643 and $1548 \mathrm{~cm}^{-1}$, respectively. Figure 5 shows the IR absorption curves around the amides I and II with the parameters being the $\mathrm{N} / \mathrm{P}$ ratios of $10 / 0,9 / 1,7 / 3$, and $5 / 5$. The intermediate compositions exhibit respective peaks intermediate between the pure components. As the respective bands of PPTA and nylon 6 are located too close to discriminate in each

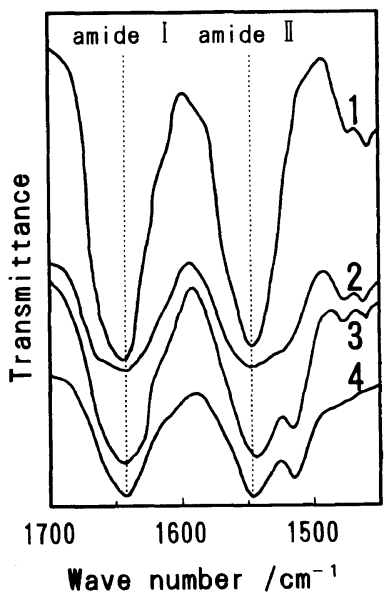

Figure 5. FTIR spectra in the region of amide $I$ and amide II $\left(1500-1700 \mathrm{~cm}^{-1}\right)$ for various composition using Ny6-A: $\mathrm{N} / \mathrm{P}=$ (1) $10 / 0$, (2) $9 / 1$, (3) $7 / 3$, and (4) $5 / 5$. region of the amide I and II bands, a more detailed discussion will not be made here.

The C-H stretching bands of $2867 \mathrm{~cm}^{-1}$ (symmetric oscillation) and $2952 \mathrm{~cm}^{-1}$ (asymmetric oscillation) are only found in nylon 6 and absent in PPTA. The results of various $\mathrm{N} / \mathrm{P}$ ratios are apparently only dependent on the nylon 6 fraction and the results are not shown here.

In summary, the $\mathrm{MC}$ of the Ny6-C sample with $M W=2.6 \times 10^{5}$ shows a single sharp peak of $\mathrm{N}-\mathrm{H}$ stretching at $\mathrm{N} / \mathrm{P}=7 / 3$ and the phase separation of nylon 6 can not be detected. The crystalline band of nylon 6 indicates the extent of dissolution of nylon 6 in PPTA for the MC of Ny6-C which is stronger than those of Ny6-A and $\mathrm{B}$ even at $\mathrm{N} / \mathrm{P}=9 / 1$.

\section{Differential Scanning Calorimetry}

Figure 6 exhibits the crystallization exotherms and the melting endotherms of the $\mathrm{MC}$ of Ny6-C as a function of compositions. In the systems of $\mathrm{N} / \mathrm{P}=1 / 9$ and $5 / 5$, the crystallization temperature, $T_{\mathrm{c}}$, and the melting temperature, $T_{\mathrm{m}}$, can not be detected. This result accords well with the absence of the crystallization band of nylon 6 in IR spectroscopy. In Figure 7, $T_{\mathrm{c}}, T_{\mathrm{m}}$, and the heat of fusion of nylon 6 crystal are shown as a function of composition of PPTA at various

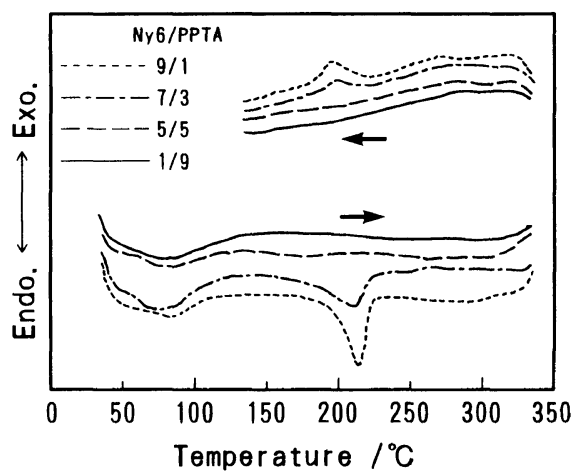

Figure 6. DSC crystallization exotherms and melting endotherms for various compositions of nylon 6/PPTA. Nylon 6 of molecular weight of $2.6 \times 10^{5}$ (Ny6-C) is used. 

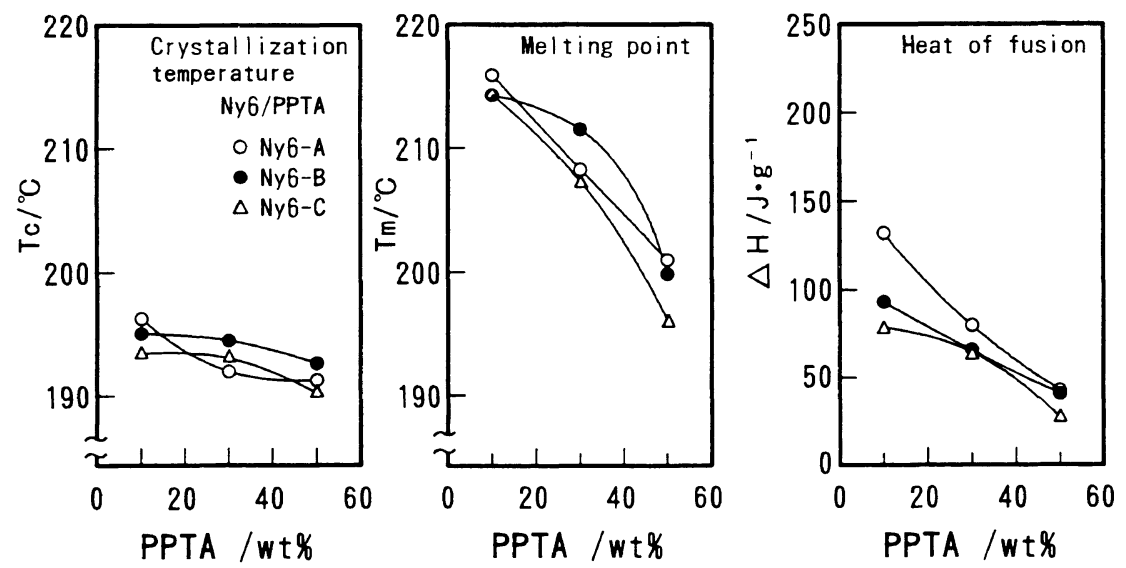

Figure 7. Variations of $T_{\mathrm{c}}, T_{\mathrm{m}}$, and heat of fusion with various compositions of nylon 6/PPTA, using different molecular weight of nylon 6 .

levels of molecular weight. $T_{\mathrm{c}}$ has no remarkable variation in the presence of PPTA. The slight decrease of $T_{\mathrm{c}}$ means that the crystallization of nylon 6 is accelerated at the interface with PPTA. This phenomenon was indicated in our previous paper. ${ }^{1}$ The effect of molecular weight of nylon 6 is negligible. It is reasonable explanation for this result that the chain segments associated with crystallization are not so long and the length of network chain between entanglements is not related to crystallization process as far as concerned with the detection of $T_{\mathrm{c}}$ by DSC method. The morphology of the texture as a whole will be affected by the entanglements in a long term observation.

$T_{\mathrm{m}}$ is slightly influenced by molecular weight. The increased molecular weight decreases $T_{\mathrm{m}}$. In the system of MC using Ny6-C, $T_{\mathrm{m}}$ is lowered due to the interruption of crystallization which forms the imperfect, defect-including crystals of nylon 6 in the region of nylon 6 content over $50 \%$. The increase in the PPTA content increases the crystal imperfection of nylon 6 .

Striking effect of molecular weight is found in the heat of fusion of nylon 6 in the MC system (the discussion is limited to the nylon 6 content larger than $50 \mathrm{wt} \%$ ). The increase of molecular weight lowers the heat of fusion of nylon 6 in the MC. The extent of crystallization of nylon 6 in Ny6-C composite is decreased due to the depressed diffusion rate of nylon 6 molecules caused by the increased internal viscosity of the melt of Ny6-C. This explains the order of heat of fusion in the MCs of Ny6-A and B.

It seems to require another explanation for the decrease in heat of fusion with increasing PPTA fraction. The dissociation energy of hydrogen-bonds between nylon 6 molecules by themselves is larger than the cross-hydrogen bond energy between the amide groups of PPTA and nylon 6. The dissolution of nylon 6 into PPTA, as $\mathrm{Kyu}$ and others ${ }^{2}$ indicated its possibility, gives rise to cross hydrogenbonding which lowers the heat of fusion. The results of FTIR also supports this explanation.

\section{$X$-Ray Diffraction}

Figures 8(a) and (b) show the X-ray diffraction intensity curves for (a) the MC of Ny6-A at various component ratios, and (b) the $\mathrm{MC}$ of Ny6-C. Curves 1, 2, 3, and 4 in both figures are for the MCs with the composition ratios of $\mathrm{N} / \mathrm{P}$ being $9 / 1,7 / 3,5 / 5$, and $1 / 9$, respectively. With increasing PPTA content, the major diffraction peaks characteristic of nylon 6 crystal located at the scattering angles 

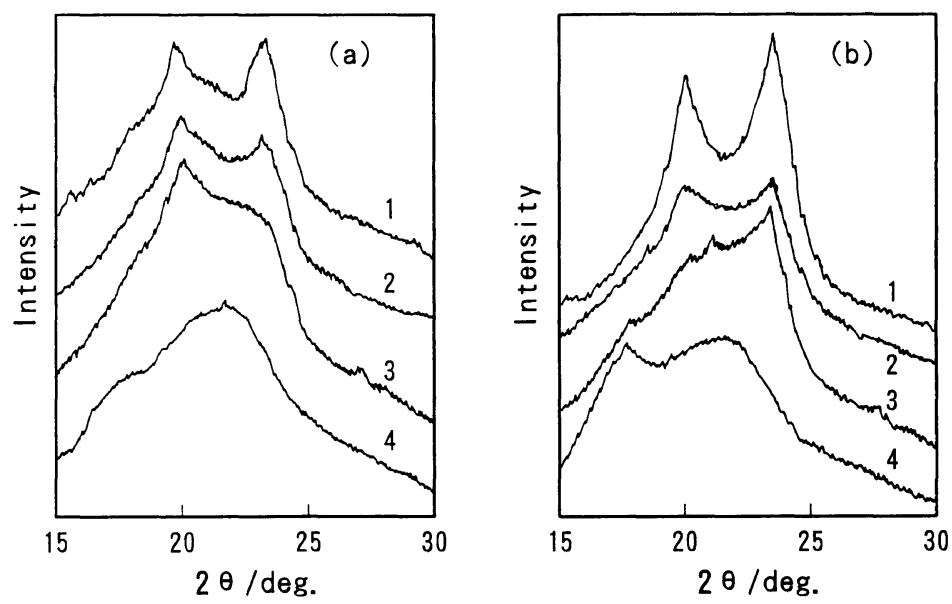

Figure 8(a) and (b). X-Ray diffraction intensity curves for the hot-pressed films of the composites: (a) the composite using Ny6-A; (b) Ny6-C. The ratios of N/P are (1) 9/1, (2) 7/3, (3) 5/5, and (4) 1/9.

of $20.1^{\circ}$ and $23.9^{\circ}$ are decreased and finally change to amorphous halo for the $\mathrm{N} / \mathrm{P}$ ratio of $1 / 9$. The disappearance of IR crystalline band and the crystallization and melting peaks in the DSC curves accord with this observation. As the MC samples are once hot-pressed at $240^{\circ} \mathrm{C}$ above the melting temperature of nylon 6 , the relative intensities vary due to variations in the orientation of crystal planes. Actually, the X-ray diffraction diagram taken by irradiating the beam along the edge direction shows uniplanar orientation of PPTA to the sheet surface, although the diffractions are largely diffused. The mechanical strength of film was improved after hot-pressing, indicating the orientation variation.

Broad peak or shoulder is found at $17.8^{\circ}$ for the composition ratios of $5 / 5$ and $1 / 9$. This scattering angle is characteristic of PPTA crystal. It might be said that nylon 6 molecules are almost completely dissolved into PPTA forming the cross-hydrogen bonding but the PPTA molecules still unsolvated with nylon 6 remain in the system, which form very thin microfibrils of PPTA incapable of detection by optical microscope. From this point of view, detailed reinspection of the single sharp peak of FTIR at $3300 \mathrm{~cm}^{-1}$ shown in
Figure 3 shows a small shoulder around $3329 \mathrm{~cm}^{-1}$ which is assigned to the $\mathrm{N}-\mathrm{H}$ stretching of PPTA.

\section{Stress-Strain Behavior}

Figures 9(a), (b), and (c) show the stressstrain curves for the MCs using Ny6-A, B, and $C$, respectively. In each figure, the $N / P$ ratios of $9 / 1,7 / 3,5 / 5$, and $1 / 9$ are denoted. With decreasing $\mathrm{N} / \mathrm{P}$ ratio, the modulus, strength and yield stress are increased. In general, the elongation-at-break tends to decrease with increasing PPTA fraction. Figure 10 shows a plot of tensile modulus versus wt $\%$ of nylon 6 for the MCs of Ny6-A, B, and C. Figure 11 shows a plot of tensile strength versus wt $\%$ of nylon 6 for three kinds of molecular weight of nylon 6 . The highest modulus of $11.8 \mathrm{GPa}$ and the strength of $170 \mathrm{MPa}$ are achieved in a hot-pressed film of MC of Ny6-C/PPTA $=1 / 9$.

The result supports the effective reinforcement of PPTA molecules to the ductile matrix of nylon 6. In this paper, the molecular weight of PPTA was kept at $5.0 \times 10^{4}$. A large difference in stress-strain behavior, for example, between Ny6-A and Ny6-C molecular composites is definitely ascribed to the degree of dispersion of PPTA molecules in nylon 6 

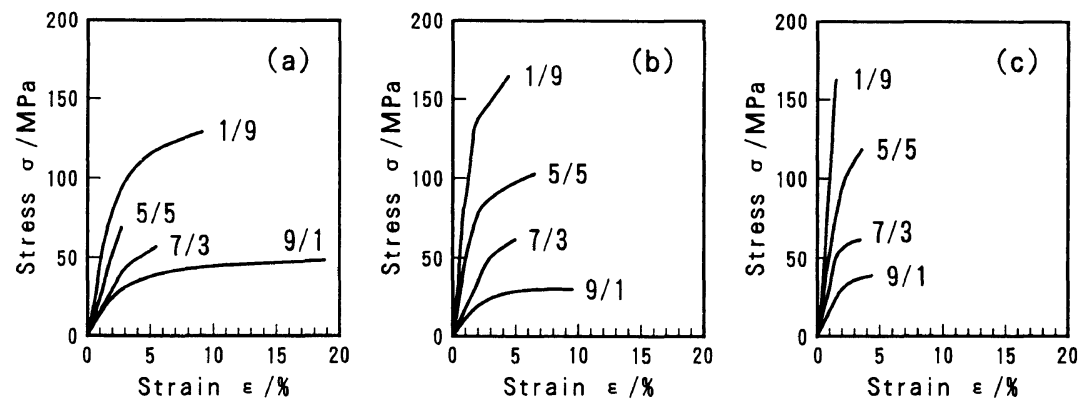

Figure 9(a), (b), and (c). Stress-strain curves for the hot-pressed films of the composites using (a) Ny6-A, (b) Ny6-B, and (c) Ny6-C. The compositions of N/P are indicated in the figure.

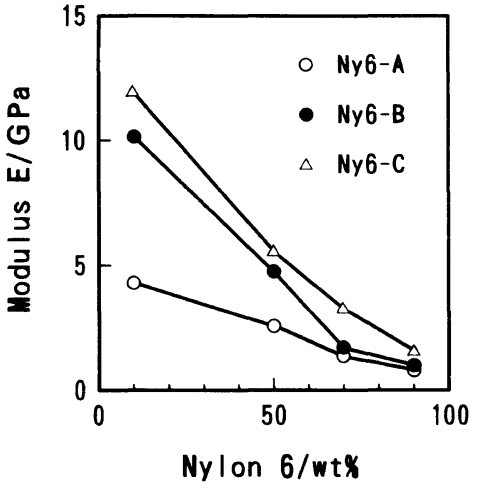

Figure 10. Tensile modulus vs. weight fraction of nylon 6 for the hot-pressed composites using different molecular weight of nylon 6.

matrix. The tendency to yielding is clearly seen in the MC of Ny6-A, whereas in the MC of Ny6-C, the ductility has almost disappeared and stiff and brittle nature is conspicuous. If compared at the same level of PPTA content, the molecularly dispersed PPTA, which was proved by the FTIR, thermal and X-ray diffraction, has an effectiveness in reinforcement with two or three times of modulus and strength of the Ny6-A composite. As reported in our previous paper, ${ }^{1}$ the state of dispersion of PPTA is in microfibrillar texture in conventional nylon 6 when the MC was hot-pressed at $260^{\circ} \mathrm{C}$. The MC of Ny6-A/PPTA in this paper is conceived to be in the same state as mentioned above. The PPTA molecules tend to associate and form microfibrils. The aspect

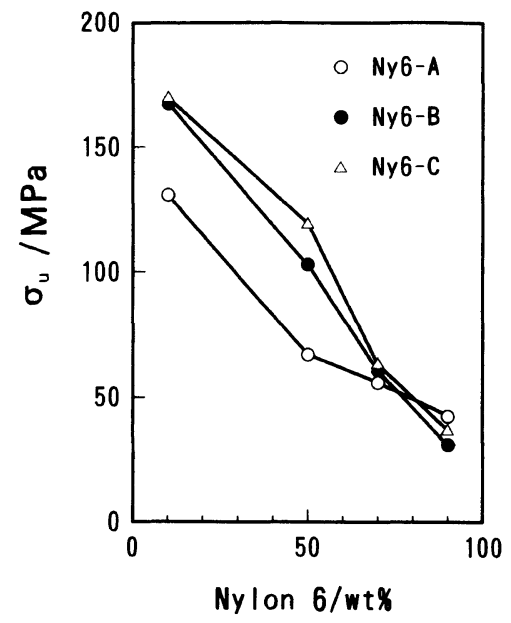

Figure 11. Tensile strength $v s$. weight fraction of nylon 6 for the hot-pressed composites using different molecular weight of nylon 6 .

ratio is largely decreased and the clusters of microfibrils are formed. When force is applied to such a specimen, the nylon 6 matrix lying in the space between PPTA microfibrillar clusters deforms plastically. This explains the yielding phenomenon of the $\mathrm{MC}$ of Ny6-A/PPTA. On the other hand, in the MC of Ny6-C/PPTA, large aspect ratio of PPTA molecule (534 in ideal case) is achieved and the stress-transfer through the connection between the PPTA molecules will be made most effectively. Our results are believed to prove the effectiveness of molecular composites. The mechanical properties of Ny6-A, B, and $\mathrm{C}$ do not play an important role in those 
of the MC. The extrapolation of the relationships of the modulus or strength versus nylon 6 fraction in the MC to the $100 \%$ nylon 6 gives no remarkable difference among them as seen in Figures 10 and 11.

To discuss the absolute values of modulus and strength of the MCs is difficult as far as the orientation of PPTA molecules or fibrils is unknown. The orientation in the film plane is optically isotropic but the orientation along the thickness direction is unknown. It will be useful to compare the mechanical performance of the film of the MC of nylon 6/PPTA with the straight PPTA film.

Recently Asahi Chemical Industry Company manufactured a film of straight PPTA with the trade name of Aramica. Scientific paper ${ }^{7}$ reported the process of film formation of Aramica: they started from the liquid crystalline solution of PPTA in sulfuric acid, the optically anisotropic dope was converted to the isotropic state by conditioning temperature and humidity which was coagulated to form a film and washed. After stretching uniaxially or biaxially, the film was dried in constant dimension and finally heat-treated. The Young's modulus was $12 \mathrm{GPa}$ and the strength was $450 \mathrm{MPa}$. Recent catalogue of Aramica lists the modulus of $15 \mathrm{GPa}$ and strength of $400 \mathrm{MPa}$ for random orientation in film plane. Uniplanar orientation was proved by the X-ray diffraction viewed from the film edge.

Referring to their report, the $\mathrm{N} / \mathrm{P}=1 / 9$ film is comparable to Aramica film in tensile modulus, but inferior in strength. Our film is isotropically coagulated and no stretching process is applied, although compression under hot-press is applied. In Aramica film, the orientation of PPTA molecules is effectively realized by stretching. The high modulus of our film comparable to that of Aramica will be realized by compressive force acting like biaxial stretching. The coexistence of nylon 6 partially helps the stress transfer among PPTA molecules or microfibrils. Lowered strength might be ascribed to the remaining heterogeneities caused by the manual operation in film formation. As a whole, the mechanical performance of our molecular composite film is unexpectedly successful in simple processing.

The problem is very low elongation-atbreak in case of Ny6-C/PPTA. Yield phenomenon is entirely lost for the $1 / 9$ composite film using Ny6-C. Nylon 6 as a matrix can not sustain the stress after the break of connectivity of PPTA molecules (in the case of Ny6-C/PPTA). As a method of removing such disadvantages, the block copolymerization has already been reported ${ }^{1}$ to be effective. The improvement of stress transfer between the PPTA molecules and nylon 6 matrix has been realized effectively with this method. Detailed report will be made in the future.

In this paper, the effect of molecular weight of matrix polymer on the phase separation of nylon 6 at the temperatures above the melting point of nylon 6 is the main subject and it was concluded that only unusually large molecular weight of nylon 6 is effective in avoiding the phase separation of nylon 6 and keeping the state of molecular dispersion of PPTA in the composite. The mechanical properties are distinctly improved by this method. The expectation of molecular composite for the future is suggested with this results.

Acknowledgments. This work was partly supported by a Grant-in-Aid for Scientific Research from the Ministry of Education, Science, and Culture of Japan. The authors also express our sincere thanks to Dr. Chiaki Tanaka of Toray Company for supplying specifically prepared nylon 6 samples with various molecular weight and to Asahi Chemical Industry Company for supplying as-polymerized PPTA sample with molecular weight of $5 \times 10^{4}$. 


\section{REFERENCES}

1. M. Takayanagi, T. Ogata, M. Morikawa, and T. Kai, J. Macromol. Sci., Phys., B17(4), 591 (1980).

2. T. Kyu, T-Ing Chen, H-Sung Park, and J. L. White, J. Appl. Polym. Sci., 37, 201 (1989).

3. P. K. Kim, C. Chang, and S. L. Hsu, Polymer, 27, 34 (1986).
4. I. Sandeman and A. Keller, J. Polym. Sci., 19, 401 (1956).

5. H. W. Starkweather, Jr. and R. E. Moynihan, J. Polym. Sci., 22, 363 (1956).

6. J. L. Koenig and M. C. Agboatwalla, J. Macromol. Sci., Phys., B2(3), 391 (1968).

7. T. Fujita, T. Fujiwara, E. Sato, K.Nagasawa, and T. Amano, Polym. Eng. Sci., 29, 1237 (1989). 